\title{
A Case of KiKuchi-Fujimoto Disease/Histiocytic Necrotizing Lymphadenitis in 25 years old African American Female
}

\author{
Jasper X Zheng ${ }^{1}$, Yingxian Liü ${ }^{2 *}$ and Armand Asarian ${ }^{3}$ \\ ${ }^{1}$ St George University School of Medicine, University Centre Grenada, Grenada \\ ${ }^{2}$ Department of Pathology, The Brooklyn Hospital, NY \\ ${ }^{3}$ Department of Surgery, The Brooklyn Hospital, $N Y$
}

*Corresponding author: Yngxian Liu, Department of Pathology, The Brooklyn Hospital, NY.

To Cite This Article: Jasper X Zheng. A Case of KiKuchi-Fujimoto Disease/Histiocytic Necrotizing Lymphadenitis in 25 years old African American Female. Am J Biomed Sci \& Res. 2019 - 5(2). AJBSR.MS.ID.000893. DOI: 10.34297/AJBSR.2019.05.000893.

Received: 盋September 05, 2019; Published: 此 September 16, 2019

\begin{abstract}
Kikuchi-Fujimoto disease, also known as histiocytic necrotizing lymphadenitis, is a self-limiting condition that primarily affects adult females less than the age of 40 . The characteristic clinical presentation of Kikuchi-Fujimoto disease is recurrent fevers, benign lymphadenopathy, and other systemic symptoms. The etiology of this syndrome is still unclear. Pathogenesis, according to clinical presentation and histological changes, suggests an immune response with the involvement of $\mathrm{T}$ cells and histiocytes. It has been proposed that Kikuchi-Fujimoto disease progression involves three distinct phases: proliferative, necrotizing, and xanthomatous phase. The histology of the involved lymph node demonstrates paracortical expansion of histiocytes, plasmacytoid dendritic cells, and CD8+ T cells. Moreover, areas of apoptotic necrosis are present in addition to characteristic absence of neutrophils. No specific laboratory tests currently exist that can diagnose Kikuchi-Fujimoto disease directly. Instead, the diagnosis requires histopathological and immunohistochemical examination in order to exclude other diseases with entirely different treatment course. Exclusion of lymphoma and systemic lupus erythematosus is imperative to avoid unnecessary and detrimental treatment. Treatment of Kikuchi disease involves only supportive measures because independent spontaneous resolution of this disease occurs within a 4-month period.
\end{abstract}

\section{Introduction}

Kikuchi-Fujimoto disease, also known as histiocytic necrotizing lymphadenitis, was first simultaneously described in 1972 by Kikuchi and Fujimoto [1,2]. Kikuchi disease has been described in both genders. Overall, there is a higher incidence in female patients. The reported age range of Kikuchi-Fujimoto disease is from 6 to 80 years with majority of the patients under the age of 40 years. The mean age at presentation in United States is 30 years of age. Even though this condition was originally reported mainly in young Japanese females, the ethnic distribution of Kikuchi disease in United States include a variety of ethnic background [3-5]. Despite its benign lymphadenopathy and self-limited clinical course, Kikuchi-Fujimoto disease requires vigilant examination in order to exclude conditions with overlapping clinical and histological features that necessitates different and more aggressive therapy.

\section{Case Report}

25 years old African American female present with 1-month duration of daily recurrent episodes of pyrexia (102 F) with painful left axilla lump. The left axilla lump was soft, tender, and mobile. Pa tient denies having fatigue, night sweats, shortness of breath, chest pain, nausea, vomiting, and diarrhea. Patient has had a decrease in appetite during the month which has led to a $10 \mathrm{lbs}$. weight loss. Patient felt dizzy at times and experienced loss of consciousness of short duration. Patient has also experienced a few days of dry cough.

Patient was prescribed Sulfamethoxazole/Trimethoprim and Cephalexin for a week. Shortly after, patient developed a rash. Drug allergy was suspected. Thus, patient was switched to clindamycin for 7 days. Without improvement, patient was sent to emergency department and had a septic workup with negative results for sepsis. Chest x-ray results were also negative.

Subsequent left axilla lymph node biopsy was performed due to suspicion of lymphoma. Gross examination of the left axillary lymph node biopsy demonstrated fragments of tan white tissue measuring $0.3 \times 0.2 \times 0.1 \mathrm{~cm}$ in dimension.

Microscopic examination of the ultrasound guided needle biopsy specimen demonstrates lymph node tissue with preserved fol- 
licular pattern and area of necrosis. Further immunohistochemical stains demonstrate CD20 and CD45 positive B-cells in follicular pattern; CD3, CD5 and BCL-2 positive T-cells in parafollicular pattern; scattered CD138 positive plasma cells; and some CD68 positive histiocytes (Figure 1). KI-67 labeling index is approximately $20 \%$. CD10, CD15, CD30, and AE 1/3 had negative immunohistochemical staining result.

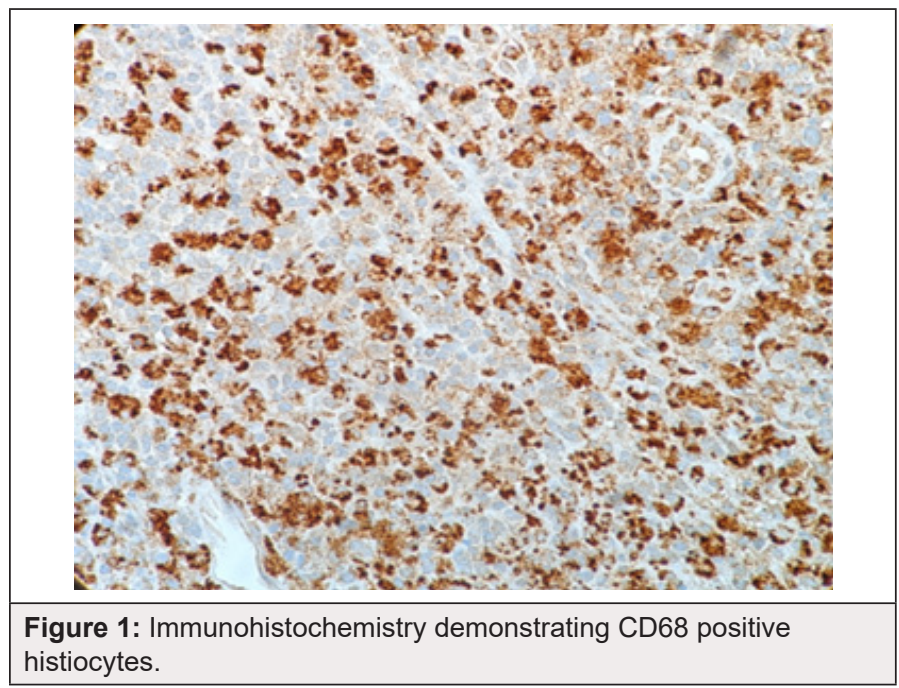

The gender, age, symptoms, histological characteristics, and immunohistochemical profile supports the diagnosis of Kikuchi-Fujimoto disease.

\section{Discussion}

Low grade fever and painless moderate sized cervical lymphadenopathy in a previously well young woman is the most common clinical manifestation of Kikuchi-Fujimoto disease [3,6]. According to Bosch et al, patients may also experience painful lymphadenopathy. The affected lymph node size can vary from $0.5-4 \mathrm{~cm}$ with rare occurrence of enlarged lymph nodes greater than $6 \mathrm{~cm} .30 \%$ to $50 \%$ of patients with Kikuchi disease can present with symptoms of upper respiratory infection, weight loss, nausea, vomiting, sore throat, and night sweats [7]. According to the retrospective literature review by Kucukardali et al. on 244 cases of Kikuchi-Fujimoto disease, the most common signs and symptoms are and not limited to: lymphadenopathy (100\%), fever (35\%), rash (10\%), arthritis (7\%), fatigue (7\%), hepatosplenomegaly (3\%) [8].

Due to unspecific laboratory and imaging characteristics, definitive diagnosis of Kikuchi-Fujimoto disease is centered on excisional lymph node biopsy and subsequent histopathological assessment $[9,10]$. Despite the self-limited nature of this syndrome, excisional lymph node biopsy is necessary in order to exclude clinically similar conditions requiring further aggressive therapy [7].

In terms of histology, the affected lymph nodes demonstrate focal, well-circumscribed, paracortical necrotizing changes. High power view of the necrotic foci demonstrates areas of karyorrhexis, pyknosis, karyolysis in addition to scattered fibrin deposits with collections of the crescentic histiocytes (Figure 2\&3). Plasmacytoid dendritic cells are often found clustered at the margins of the necrotic foci [9,11-13]. Scanty plasma cell will be seen if not absent. In addition, the absence of neutrophils and eosinophils is a distinguished characteristic of this syndrome. A mixture of large to small lymphocytes are also present. The numerous presences of large lymphocytes with immunoblastic morphology in a background of karyorrhectic debris and scattered tangible body of macrophages, can often mislead the diagnosis towards high-grade lymphoma [9,11-13]. These necrotic foci may be single or multiple with varying degree of necrosis that differ from case to case $[7,9,11-13]$.



Figure 2: High-power view showing the boundary between an area of karyorrhexis/pyknosis and an area of karyolysis ( $\mathrm{H} \& \mathrm{E}$, original magnification $\times 100$ )



Figure 3: Crescentic Histiocytes; background of apoptotic necrosis with abundant karyorrhectic debris (H\&E, original magnification $\times 600$ )

As the disease progress, the histological appearance will also change accordingly. The early proliferative phase is characterized with follicular hyperplasia and paracortical proliferation of T-cells, B-cells, plasmacytoid monocytes, lymphocytes, and histocytes (Figure 4). Numerous apoptosis in the background of this proliferative picture is also a distinguishing feature. As the disease progress to the later necrotizing phase, the histological appearance will progressively change to show necrosis with the absence of neutrophil 
infiltrate. The presences of numerous crescentic histocytes with or without phagocytosed debris is a distinct feature of the necrotizing phase as well as Kikuchi-Fujimoto disease itself. Finally, with or without necrotic foci, the presence of foamy histocytes indicate the categorization of xanthomatous phase [9,11-13].

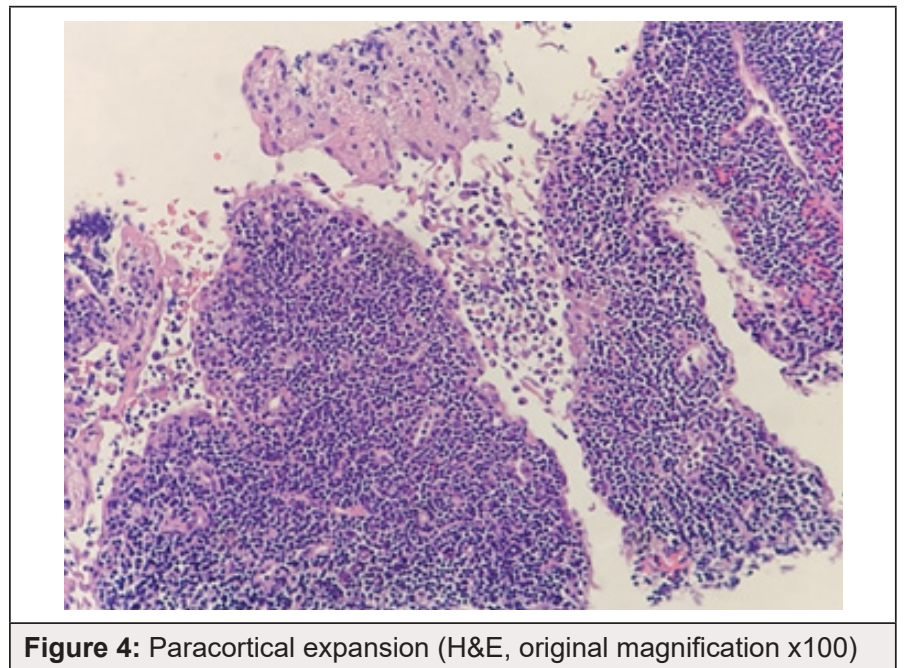

Histological differential diagnosis primarily consists of lymphoid malignancy, autoimmune associated lymphadenopathy, and infectious diseases. Systematic exclusion of these entities during the diagnosis of Kikuchi disease is imperative because each of these entities requires disease specific management [9,11-13].

As mentioned previously, the abundance of plasmacytoid dendritic cells and activated T-cell closely mimics the appearance of malignant lymphoma. Necrosis with absence of neutrophil is characteristic of Kikuchi disease. In comparison, necrosis associated with Hodgkin's lymphoma usually includes numerous neutrophils and eosinophils surrounding large atypical Reed-Sternberg variant cells [12]. These large atypical cells are immunohistochemically positive for CD15, CD30 and PAX-5. The proliferative phase aggregation of immunoblastic proliferation and the necrotic phase plasmacytoid dendritic cells of Kikuchi Fujimoto disease closely resembles the histological presentation of anaplastic large cell lymphoma $[9,12]$. With the assistance of immunohistology staining, large cell lymphoma can be excluded with the absence of CD30 expression. Similarly, B-cell linage of large cell lymphoma can be excluded with lack of CD20, CD79a, or PAX5 expression. [9,12]. Flow cytometrical analysis may be utilized in difficult and confusing cases.

It is a difficult task to differentiate Kikuchi Fujimoto disease from systemic lupus erythematosus due to near resemblance and occasional identical histological presentation. Useful differentiating features that favors Kikuchi disease includes abundance of CD8 positive cytotoxic $\mathrm{T}$ cells around the necrotic areas, scanty plasma cell presence, and absence of neutrophils and eosinophils $[7,14]$. Differentiating features that favors SLE/autoimmune disease includes the presence of hematoxylin bodies, Azzopardi phenomenon, reactive follicular hyperplasia, scanty cytotoxic t-cells, abundance of plasma cells, and capsular and pericapsular inflammation $[7,14]$. Due to close histological resemblance and morphology of these two diseases, it is of diagnostic importance, especially in ambiguous situations, to also review patient laboratory data and clinical history [9,11-13]. Infection induced adenopathy will present similar histological features that closely resembles Kikuchi disease. On histological examination, Kikuchi Fujimoto disease can be differentiated from viral lymphadenitis with significant histiocytic infiltrate while lacking in neutrophil aggregation, CD4+ T cell presence, and plasma cell proliferation [12]. Furthermore, due to similar clinical presentation and radiological findings, tuberculosis requires careful differentiation from Kikuchi Fujimoto disease. To differentiate, Kikuchi Fujimoto disease lacks the presence of epithelioid histiocytes, multinucleated giant cells, and granuloma formation found in necrotizing granulomatous lymphadenitis of tuberculosis $[11,15]$.

Kikuchi Fujimoto disease is a self-limiting syndrome. Signs and symptoms usually take four months for full resolution. Recurrence rate is low at around 3-4\% [16]. Clinicians should be aware that on rare occasions Kikuchi Fujimoto disease is associated with aggressive life-threatening conditions that include disseminated intravascular coagulation, hemophagocytic syndrome, pulmonary hemorrhage, severe infection, and acute heart failure [16]. Therapy is mainly focused on alleviating symptoms which include relief of fever with antipyretics and reduction of lymph node tenderness with analgesics $[9,12,13]$.

\section{Conclusion}

Kikuchi disease, despite its benign lymphadenopathy and self-limited clinical course, requires vigilant examination in order to exclude conditions with overlapping clinical and histological features requiring further aggressive therapy.

\section{References}

1. Kikuchi M (1972) Lymphadenitis showing focal reticulum cell hyperplasia with nuclear debris and phagocytosis. Nippon Ketsueki Gakkai Zasshi 35: 378-380.

2. Fujimoto Y, Kozima Y, Hamaguchi K (1972) Cervical necrotizing lymphadenitis: a new clinicopathological agent. Naika 20: 920-927.

3. Dorfman RF, Berry GJ (1988) Kikuchi's histiocytic necrotizing lymphadenitis: an analysis of 108 cases with emphasis on differential diagnosis. Semin Diagn Pathol 5(4): 329-345.

4. Lee KY, Yeon YH, Lee BC (2004) Kikuchi-Fujimoto disease with prolonged fever in children. Pediatrics 114: e752.

5. Payne JH, Evans M, Gerrard MP (2003) Kikuchi-Fujimoto disease: a rare but important cause of lymphadenopathy. Acta Paediatr 92: 261.

6. Asano S, Akaike Y, Jinnouchi H, Muramatsu T, Wakasa H (1990) Necrotizing lymphadenitis: a review of clinicopathological, immunohistochemical and ultrastructural studies. Hematol Oncol 8(5): 251-260.

7. Bosch X, A Guilabert R Miquel, E Campo (2004) Enigmatic Kikuchi-Fujimoto disease: a comprehensive review. Am J Clin Pathol 122(1): 141152.

8. Kucukardali Y, Solmazgul E, Kunter E, Oncul O, et al. (2007) Kikuchi-Fujimoto Disease: analysis of 244 cases. Clin Rheumatol 26(1): 50-54. 
9. Deaver D, Horna P, Cualing H, Sokol L (2014) Pathogenesis, diagnosis, and management of Kikuchi-Fujimoto disease. Cancer Control 21(4) 313-321.

10. Das DK, Mallik MK, Dashti HA, et al. (2013) Kikuchi-Fujimoto disease in fine-needle aspiration smears: a clinico-cytologic study of 76 cases of KFD and 684 cases of reactive hyperplasia of the lymph node. Diagn Cytopathol 41(4): 288-295.

11. Pileri SA, F Facchetti, S Ascani, Sabattini E, Poggi S, et al. (2001) Myeloperoxidase expression by histiocytes in Kikuchi's and Kikuchi-like lymphadenopathy. Am J Pathol 159(3): 915-924.

12. Charles Blake Hutchinson, Endi Wang (2010) Kikuchi-Fujimoto Disease. Archives of Pathology \& Laboratory Medicine 134(2): 289-293.
13. Shahid S, Alam SH, Hadley I (2018) An Unusual Presentation of Kikuchi-Fujimoto Disease with Recurrent Subdural Effusion. Cureus 10(3): e2302.

14. Baenas DF, Diehl FA, Haye Salinas MJ, Riva V, Diller A, et al. (2016) Kikuchi-Fujimoto disease and systemic lupus erythematosus. Int Med Case Rep J 9: 163-167.

15. Yu SC, Chen CN, Huang HI, Hsiao TY, Yang TL4et al. (2014) Diagnosis of Kikuchi-Fujimoto disease: a comparison between open biopsy and minimally invasive ultrasound-guided core biopsy. PLoS One 9(5): e95886.

16. Singh JM, Shermetaro CB (2019) Kikuchi-Fujimoto Disease in Michigan: A Rare Case Report and Review of the Literature. Clin Med Insights Ear Nose Throat 12: 1179550619828680. 